Independent Submission

Request for Comments: 7093

Category: Informational

ISSN : 2070-1721
S. Turner

IECA

S. Kent

BBN

J. Manger

Telstra

December 2013

\title{
Additional Methods for Generating Key Identifiers Values
}

Abstract

This document specifies additional example methods for generating Key Identifier values for use in the AKI (Authority Key Identifier) and SKI (Subject Key Identifier) certificate extensions.

Status of This Memo

This document is not an Internet Standards Track specification; it is published for informational purposes.

This is a contribution to the RFC Series, independently of any other RFC stream. The RFC Editor has chosen to publish this document at its discretion and makes no statement about its value for implementation or deployment. Documents approved for publication by the RFC Editor are not a candidate for any level of Internet Standard; see Section 2 of RFC 5741.

Information about the current status of this document, any errata, and how to provide feedback on it may be obtained at http://www.rfc-editor.org/info/rfc7093.

Copyright Notice

Copyright (c) 2013 IETF Trust and the persons identified as the document authors. All rights reserved.

This document is subject to BCP 78 and the IETF Trust's Legal Provisions Relating to IETF Documents (http://trustee.ietf.org/license-info) in effect on the date of publication of this document. Please review these documents carefully, as they describe your rights and restrictions with respect to this document. 


\section{Introduction}

[RFC5280] defines the AKI (Authority Key Identifier) and SKI (Subject Key Identifier) certificate extensions. [RFC5280] describes two example mechanisms for generating AKI and SKI values: a 160-bit SHA-1 (Secure Hash Algorithm) hash of the public key and a four-bit type field with the value 0100 followed by the least significant 60 bits of the SHA-1 hash. Both of these mechanisms were designed to not be critical to security. This document defines three additional mechanisms for generating Key Identifier values using SHA-256, SHA-384, and SHA-512 [SHS] that are similar to those examples defined in [RFC5280] as well as one based on hashing the certificate's Subject Public Key Info field.

2. Additional Methods for Generating Key Identifiers

[RFC5280] specifies two examples for generating key identifiers from public keys. Four additional mechanisms are as follows:

1) The keyIdentifier is composed of the leftmost 160-bits of the SHA-256 hash of the value of the BIT STRING subjectPublickey (excluding the tag, length, and number of unused bits).

2) The keyIdentifier is composed of the leftmost 160-bits of the SHA-384 hash of the value of the BIT STRING subjectPublickey (excluding the tag, length, and number of unused bits).

3) The keyIdentifier is composed of the leftmost 160-bits of the SHA-512 hash of the value of the BIT STRING subjectPublickey (excluding the tag, length, and number of unused bits).

4) The keyIdentifier is composed of the hash of the DER encoding of the subjectPublickeyInfo value. 


\section{Examples}

This section provides some examples. The keys and SKIs are presented in hexadecimal (two hex digits per byte).

Given the following DER-encoded SubjectPublickeyInfo value holding an P-256 ECDSA (Elliptic Curve Digital Signature Algorithm) key:

$30 \quad 59$

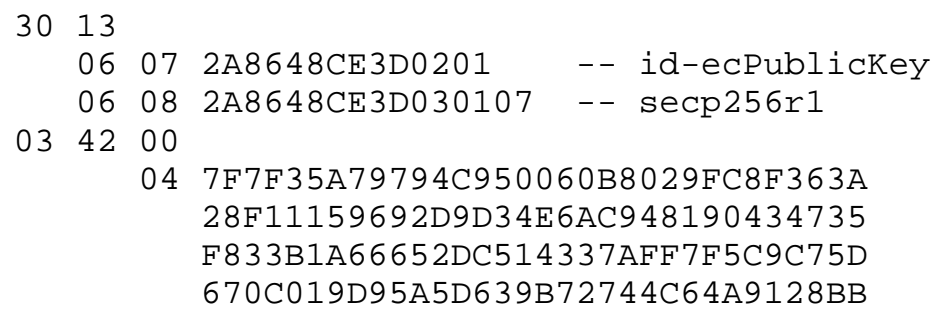

The SHA-256 hash of the 65 bytes $047 F 7 F \ldots B B$ is:

BF37B3E5808FD 46D54B28E846311BCCE1CAD2E1A62AA90 92EF3EFB3F11451F 44

The SHA-1 hash of these 65 bytes is:

6FEF 9162C0A3F2E7608956D41C37DA0C8E87F0AE

The SHA-256 hash of the 91 bytes $305930 \ldots$ BB is:

6D20896AB8BD833B6B66554BD59B20225D8A75A296088148399D7BF 763D57405

Using method 1 from Section 2, the subjectKeyIdentifier would be:

$301 \mathrm{D}$

0603 551D0E -- id-ce-subjectKeyIdentifier

0416

0414 BF37B3E5808FD46D54B28E846311BCCE1CAD2E1A

Using method 4 from Section 2 with SHA-256 and no truncation, the subjectKeyIdentifier extensions would be:

3029

0603 551D0E -- id-ce-subjectKeyIdentifier

$04 \quad 22$

0420 6D20896AB8BD833B6B66554BD59B2022

5D8A75A296088148399D7BF763D57405 
4. Security Considerations

The security considerations of [RFC5280] apply to certificates. The security considerations of [RFC5758] apply to the hash algorithms.

While hash algorithms provide preimage resistance, second-preimage resistance, and collision resistance, none of these properties are needed for key identifiers.

5. Acknowledgements

The authors wish to thank Santosh Chokhani, Stephen Farrell, Tom Gindin, Peter Gutmann, Henry Holtz, David Kemp, Timothy Miller, Michael StJohns, Stefan Santesson, Jim Schaad, Rene Struik, Koichi Sugimoto, and Carl Wallace for taking the time to participate in the discussions about this document. The discussions resulted in numerous editorial and technical changes to the document.

6. Normative References

[RFC5280] Cooper, D., Santesson, S., Farrell, S., Boeyen, S., Housley, R., and W. Polk, "Internet X.509 Public Key Infrastructure Certificate and Certificate Revocation List (CRL) Profile", RFC 5280, May 2008 .

[RFC5758] Dang, Q., Santesson, S., Moriarty, K., Brown, D., and T. Polk, "Internet X.509 Public Key Infrastructure: Additional Algorithms and Identifiers for DSA and ECDSA", RFC 5758, January 2010 .

[SHS] National Institute of Standards and Technology (NIST), FIPS Publication 180-3: Secure Hash Standard, October 2008 . 


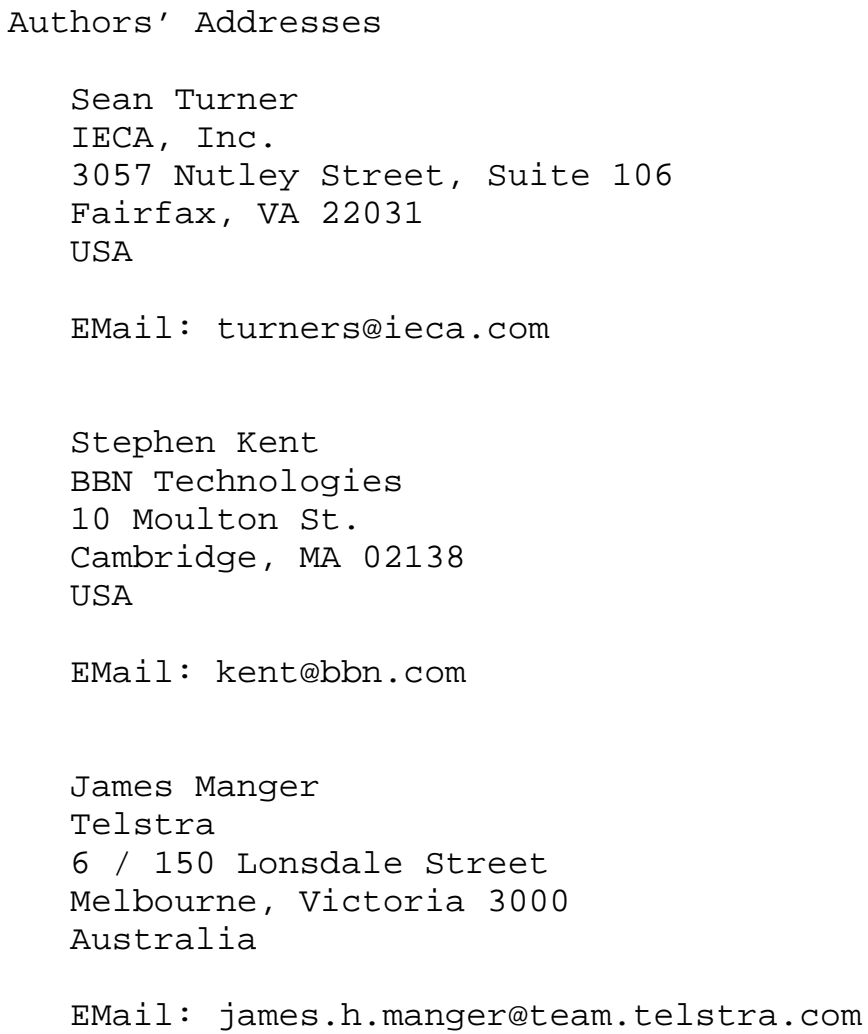

EMail: james.h.mangerateam.telstra.com 